\title{
Heterologous immunity provides a potent barrier to transplantation tolerance
}

\author{
Andrew B. Adams, ${ }^{1}$ Matthew A. Williams, ${ }^{1}$ Thomas R. Jones, ${ }^{1}$ Nozomu Shirasugi, ${ }^{1}$ \\ Megan M. Durham, ${ }^{1}$ Susan M. Kaech, ${ }^{2}$ E. John Wherry, ${ }^{2}$ Thandi Onami, ${ }^{2}$ \\ J. Gibson Lanier, ${ }^{2}$ Kenneth E. Kokko, ${ }^{1}$ Thomas C. Pearson, ${ }^{1}$ Rafi Ahmed, ${ }^{2}$ \\ and Christian P. Larsen ${ }^{1}$
}

${ }^{1}$ Emory Transplant Center and Department of Surgery and

${ }^{2}$ Emory Vaccine Center and Department of Microbiology and Immunology, Emory University School of Medicine, Atlanta, Georgia, USA

\begin{abstract}
Many strategies have been proposed to induce tolerance to transplanted tissue in rodents; however, few if any have shown equal efficacy when tested in nonhuman primate transplant models. We hypothesized that a critical distinction between specific pathogen-free mice and nonhuman primates or human patients is their acquired immune history. Here, we show that a heterologous immune response - specifically, virally induced alloreactive memory - is a potent barrier to tolerance induction. A critical threshold of memory T cells is needed to promote rejection, and CD8 "central" memory $\mathrm{T}$ cells are primarily responsible. Finally, treatment with deoxyspergualin, an inhibitor of NF- $\mathrm{KB}$ translocation, together with costimulation blockade, synergistically impairs memory $T$ cell activation and promotes antigen-specific tolerance of memory. These data offer a potential explanation for the difficulty encountered when inducing tolerance in nonhuman primates and human patients and provide insight into the signaling pathways essential for memory $\mathrm{T}$ cell activation and function.

J. Clin. Invest. 111:1887-1895 (2003). doi:10.1172/JCI200317477.
\end{abstract}

\section{Introduction}

A critical feature of the adaptive immune response is the ability to recognize previously encountered pathogens. Memory $\mathrm{T}$ cells, in contrast to naive cells, are programmed to activate quickly, with a reduced requirement for costimulatory signals. Upon re-exposure to antigen, they rapidly regain effector function and generate a swift response that controls the pathogen and prevents disease (1). Phenotypic subsets of memory $\mathrm{T}$ cells, designated "central" and "effector" memory, have recently been described with distinct cytotoxic and proliferative capacities (2-4). However, the role of these individual subpopulations during secondary immune responses in vivo remains to be determined.

It is now clear that previous immunological exposures and resultant $\mathrm{T}$ cell memory can influence the course of future immune responses to unrelated pathogens, a phenomenon termed heterologous immunity (5). Considerably

Received for publication November 26, 2002, and accepted in revised form March 11, 2003.

Address correspondence to: Christian P. Larsen, Emory Transplant Center and Department of Surgery, Suite 5105 WMB, 1639 Pierce Drive, Atlanta, Georgia 30322, USA.

Phone: (404) 727-8466; Fax: (404) 727-3660;

E-mail: clarsen@emoryhealthcare.org.

Conflict of interest: The authors have declared that no conflict of interest exists.

Nonstandard abbreviations used: Epstein-Barr virus (EBV); 15-deoxyspergualin (DSG); lymphocytic choriomeningitis virus (LCMV); vaccinia virus (VV); vesicular stomatitis virus (VSV); cytotoxic T lymphocyte (CTL); median survival time (MST). less is known regarding the effect of an individual's immune history on the response to an allogeneic tissue transplant. Although the frequency of naive T cells available to respond to any given pathogen is relatively small (approximately 1:200,000), the proportion that can directly recognize foreign $\mathrm{MHC}$ represents a substantial fraction of the total T cell repertoire (up to 1-10\%) $(6,7)$. Given this extraordinary precursor frequency and the degeneracy of $T$ cell recognition, it is not surprising that alloreactive $\mathrm{T}$ cells become activated after viral infection in experimental models (8-11). Similarly, allo-cross-reactivity for HLA-B14, -44, and -35 has been demonstrated by HLA-B8-restricted Epstein-Barr virus-specific (EBVspecific) $T$ cells in humans (12). Interestingly, one of these cross-reactive combinations (B44 $\rightarrow$ B8) was identified as an "immunogenic pairing" (i.e., reduced allograft survival) in a renal transplant registry analysis (13). Further evidence of the clinical importance of cross-reactive memory $\mathrm{T}$ cells has been provided by Heeger et al., who have shown that a higher frequency of anti-donor memory $\mathrm{T}$ cells (presumably induced by prior environmental exposures) is associated with an increased rejection rate in clinical renal transplantation (14).

A variety of strategies have been devised to promote long-term allograft acceptance without the need for chronic immunosuppression in rodent models. Unfortunately, these protocols have proven to be markedly less effective when tested in preclinical primate models or in humans. One major distinction between specific pathogen-free mice and nonhuman primates or human 
patients is the exposure of the latter to pathogens and the resultant immune history. Although many tolerance strategies, such as regimens employing $\mathrm{T}$ cell costimulation blockade, successfully target and control naive $\mathrm{T}$ cell responses, memory cells have a lower threshold for activation $(15,16)$ and may not be susceptible. In support of this, Valujskikh et al. have shown that the presence of sensitized cells prevents the beneficial effects of anti-CD40L in prolonging allograft survival (17). Thus, strategies that successfully tolerize naive cells may fail when the host has pre-existing anti-donor memory, perhaps explaining the relative inability of these regimens to promote tolerance in large-animal models. Here, we provide evidence that an individual's immune memory can profoundly influence the outcome of tolerance induction protocols even when strategies are used that uniformly induce tolerance to highly immunogenic tissues in vigorous experimental models. First, we document that viral infections can lead to the generation of alloreactive memory $\mathrm{T}$ cells, which can confer resistance to tolerance induction. The quantity of accumulated anti-donor memory is critical - that is, there is a threshold of memory cells necessary to promote rejection - and we provide evidence that $\mathrm{CD} 8^{+}$central memory $\mathrm{T}$ cells are principally responsible for rejection. Further, there is a hierarchy in susceptibility to tolerance: naive animals are uniformly sensitive to tolerance induction; recipients exposed to a single prior infection show a slightly diminished susceptibility; and "immunologically experienced" hosts, having received multiple sequential infections, are refractory to tolerance induction. Finally, we show that the immunosuppressive agent 15-deoxyspergualin (DSG), an inhibitor of NF- $\mathrm{KB}$ translocation, synergizes with costimulation blockade to promote antigen-specific tolerance of both naive and memory $\mathrm{T}$ cells. These data suggest that heterologous immunity - in particular, virally induced alloreactive memory - may be an underappreciated barrier to tolerance induction in transplantation.

\section{Methods}

Mice and viral infections. BALB/c, C57BL/6 (B6, CD45.2),

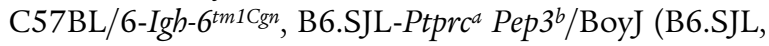
CD45.1) mice were purchased from the Jackson Laboratory (Bar Harbor, Maine, USA). To induce acute infection, mice were inoculated with $2 \times 10^{5}$ PFUs of the lymphocytic choriomeningitis virus (LCMV) Armstrong strain (intraperitoneally), $5 \times 10^{6} \mathrm{PFUs}$ of vaccinia virus (VV) (intraperitoneally), or $2 \times 10^{6} \mathrm{PFUs}$ of vesicular stomatitis virus (VSV) (intravenously). For viral rechallenge experiments, immune mice received $2 \times 10^{6} \mathrm{PFUs}$ of LCMV clone 13 (intravenously). Virus stocks were grown and quantitated as previously described (18). Infectious LCMV in serum and tissues was measured by plaque assay on Vero cell monolayers as described (18). Skin grafting and tolerance induction protocols. Full-thickness skin grafts and the mixed chimerism tolerance protocol using CTLA4-Ig (Bristol-Myers Squibb, Princeton, New Jersey, USA), anti-CD40L (Bioexpress, Lebanon, New Hampshire, USA), and busulfan was administered as previously described (19). Rapamycin $(0.4 \mathrm{mg} / \mathrm{kg} /$ day intraperitoneally; d0-6, Wyeth, Philadelphia, Pennsylvania, USA), anti-IL-2R mAb (250 $\mu \mathrm{g}$ intraperitoneally; d0,2,4,6-PC61), anti-common $\gamma$ chain $(500 \mu \mathrm{g}$ intraperitoneally; d0,2,4,6-3E12/ $4 \mathrm{G} 3$, a gift from Thomas Malek), DSG (5 $\mathrm{mg} / \mathrm{kg}$ per day intraperitoneally; and d0-6, a gift from Nippon Kayaku, Tokyo, Japan) were administered as indicated. Cell preparations and flow cytometry. Intracellular IFN- $\gamma$ expression was induced in response to 4-5 hours of ex vivo restimulation with LCMV peptides or allogeneic stimulators. In brief, splenocytes were prepared from experimental and naive animals. Responders were resuspended in cell culture media containing brefeldin (GolgiPlug, BD Pharmingen, San Diego, California, USA) and recombinant hIL-2 $(10 \mathrm{ng} / \mathrm{ml})$. To enumerate the allogeneic response, responders were plated in a 96-well plate at a 1:1 ratio $\left(1 \times 10^{6}\right.$ cells per well $)$ with stimulators (splenocyte preparation from naive animals, either allogeneic $[\mathrm{BALB} / \mathrm{c}]$ or syngeneic). To quantify the viral response, responder preparations were either incubated with viral peptides (LCMV and VSV) or with infected stimulator cells as previously described $(20,21)$. Responders alone, irrelevant peptide, or stimulation with syngeneic splenocytes were used as specificity controls. All stimulations were performed for $4-5$ hours at $37^{\circ} \mathrm{C}$. Intracellular staining for IFN- $\gamma$ was performed as per the manufacturer's instructions (Cytofix/Cytoperm kit, BD Pharmingen). Peripheral blood was analyzed by staining with the indicated fluorochrome-conjugated antibodies (BD Pharmingen), followed by red blood cell lysis and washing with a whole-blood lysis kit (R\&D Systems, Minneapolis, Minnesota, USA). Flow cytometry was performed on a FACSCalibur, and data were analyzed using Cellquest software (Becton-Dickinson, Braintree, Massachusetts, USA). Cell selections were achieved using a MACS magnetic sorting system (Miltenyi BioTec, Auburn, California, USA).

Statistical analyses. Statistical analyses were performed using a Mann-Whitney U test for skin graft survival; otherwise, a Student's $t$ test was used.

\section{Results}

Viral infections induce heterologous allospecific effector and memory responses. Although previous studies in both mice and man have documented allospecific cytotoxic $T$ lymphocyte (CTL) activity immediately after viral infection (9-11), there is little quantitative information on the retention of alloreactive memory $T$ cells after viral infections and their potential effects on transplantation. As an initial approach to determine whether prior exposure to viral pathogens might influence the response to transplanted allogeneic tissues, we studied the expansion and retention of virus-specific and alloreactive effector and memory cells during and after acute viral infections with LCMV (Armstrong strain), VV, or VSV. Each of these viruses produces an acute infection that stimulates an appropriate immune response, resulting in rapid clearance of the pathogen and the generation of a stable 
memory $\mathrm{T}$ cell population that persists for the life of the mouse $(20,22,23)$. Mice $\left(\mathrm{C} 57 \mathrm{BL} / 6, \mathrm{H}-2^{\mathrm{b}}\right)$ were infected with the individual viruses, and the number of virus-specific and alloreactive ( $\mathrm{H}-2^{\mathrm{d}}$-stimulated) IFN- $\gamma$-producing cells were measured in the effector phase (peak response) or during the memory phase (more than 6 weeks after infection) after viral clearance. For comparison, we measured the magnitude of the anti-donor $T$ cell response during and after skin allografts (Figure 1). Agematched naive control mice evaluated at similar time points never displayed detectable antiviral or anti-H- ${ }^{\mathrm{d}}$ responses (data not shown).

The number of virus-specific cells in both the effector and memory stages, as measured by intracellular staining for IFN- $\gamma$ production (Figure 1a), was consistent with prior reports for each virus $(20,21,24,25)$. Animals infected with $\mathrm{LCMV}$ or $\mathrm{VV}$ generated a readily detectable heterologous anti- $\mathrm{H}-2^{\mathrm{d}}$ response, as measured by the number of $\mathrm{T}$ cells producing IFN- $\gamma$ upon short-term, in vitro rechallenge with $\mathrm{BALB} / \mathrm{c}$ splenocytes. As expected, the virally induced heterologous anti- $\mathrm{H}-2^{\mathrm{d}}$ response was lower in magnitude than the viral-specific response (LCMV [NP396-404], Figure 1b; VV [infected syngeneic cells], data not shown) or the anti-donor response after skin graft challenge (Figure 1b), but it still represented about $1-10 \%$ of the overall antiviral response. Despite previous reports that infection with VSV elicits a crossreactive allogeneic response (anti-H-2 $\left.{ }^{\mathrm{bm} 8}\right)(9)$, we found no comparable response to a distinct haplotype $\left(\mathrm{H}-2^{\mathrm{d}}\right)$, suggesting that the magnitude of the virally induced heterologous response is dependent on the particular virus-MHC pairing being evaluated.

Donor-specific memory prevents tolerance induction. Having demonstrated that an encounter with environmental pathogens results in the generation of memory T cells with specificity for foreign MHC, we next examined whether donor-reactive memory cells could prevent tolerance induction using a protocol that induces robust, life-long deletional tolerance to fully MHC-mismatched skin grafts (19). Briefly, mice received a short course of costimulation blockade (CTLA4-Ig and antiCD40L) in addition to donor bone marrow infusion preceded by a single dose of busulfan, a stem cell-selective agent used to promote engraftment of the donor bone marrow cells. Naive mice treated with this protocol exhibit stable mixed hematopoietic chimerism (approximately 50\% donor cells in peripheral blood) and deletional donor-specific tolerance (19).

$\mathrm{B}$ cell-deficient mice were initially chosen to study the effects of anti-donor memory on tolerance induction in order to permit the study of anti-donor $\mathrm{T}$ cell memory in the absence of anti-donor antibody. Although naive B6 B cell-deficient recipients (C57BL/6-Ig $\mathrm{F}^{-/}$) receiving the tolerance protocol developed stable mixed chimerism and accepted donor-specific BALB/c skin grafts (median survival time [MST], more than 75 days) a

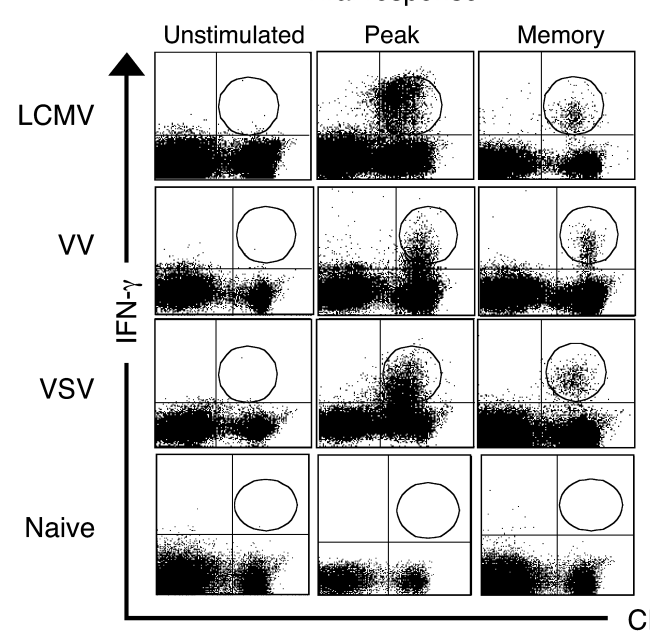

Skin graft
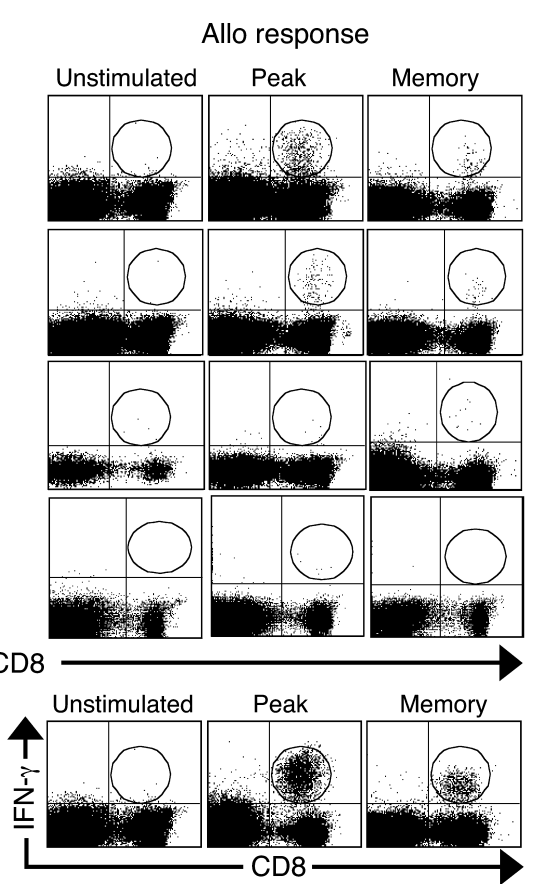

b
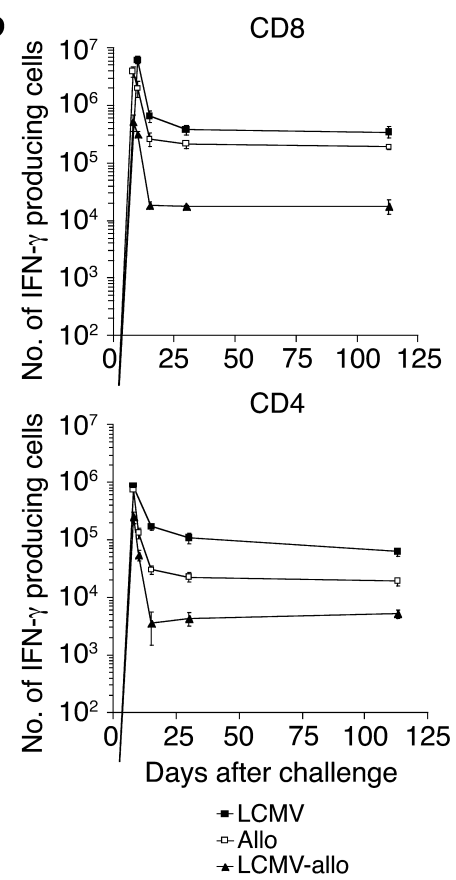

Figure 1

Analysis of viral- and allo-specific responses in vivo. B6 mice were infected with either LCMV, W, or VSV. The results are displayed for the peak of the response as well as during the memory phase (more than 6 weeks). For comparison, the response to a skin allograft is also shown. (a) Representative dot plots displaying the number of CD8 ${ }^{+}$IFN- $\gamma^{+}$T cells representing the antiviral response (LCMV-NP396-404, W-infected stimulators, VSV-N-peptide; left panels) and the corresponding heterologous allo-specific responses (right panels). (b) The number of virus-specific (NP396-404, filled squares) and virally induced, alloreactive T cells ( $\mathrm{H}-2^{\mathrm{d}}$ stimulated, filled triangles) after $\mathrm{LCMV}$ infection are shown over time. The allo-specific response after skin graft is shown for comparison $\left(\mathrm{H}-2^{\mathrm{d}}\right.$ stimulated, open squares; $\mathrm{CD}^{+}$, upper panel; $\mathrm{CD}^{+}$, lower panel). Each time point represents the average for three animals. Experiments were repeated three times with similar results. 


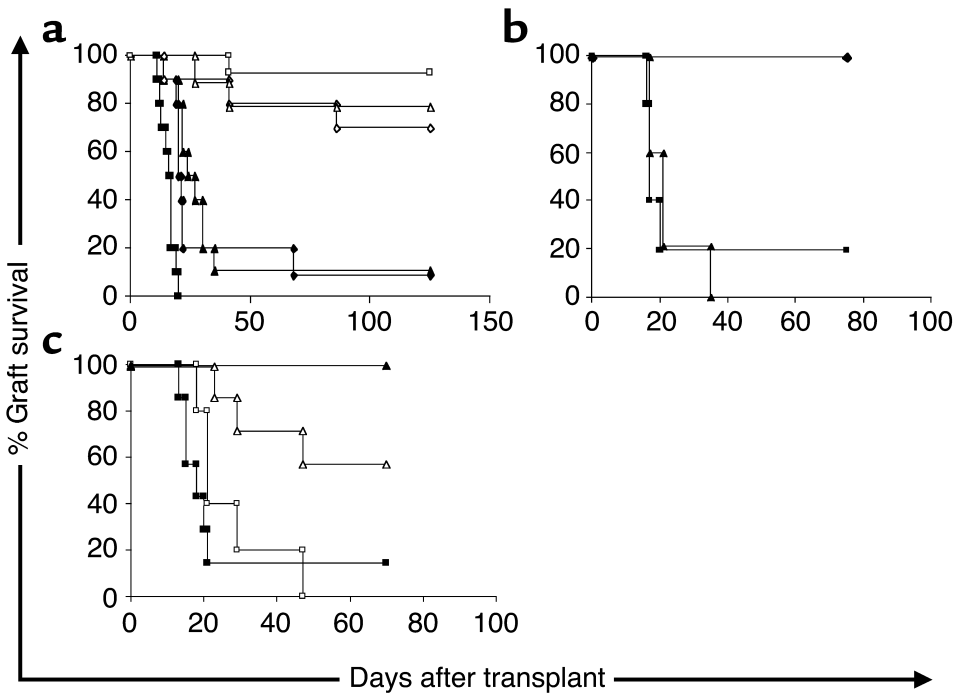

Figure 2

A critical number of $C D 8^{+}$central memory $T$ cells are needed to resist tolerance. Naive B6 mice (CD45.2) received $T$ cells from sensitized congenic B6 mice (CD45.1). (a) Titration of memory cell numbers. Naive recipients received varying doses of $T$ cells (calculated number of memory cells transferred in brackets) from sensitized hosts $\left(2 \times 10^{7}\right.$ [400,000], filled squares; $10^{7}$ [200,000], filled diamonds; $5 \times 10^{6}[100,000]$, filled triangles; $2 \times 10^{6}[40,000]$, open diamonds; $10^{6}$ [20,000], open triangles; $2 \times 10^{7}$ naive cells [none], open squares). (b) CD8 ${ }^{+}$ memory $T$ cells represent a potent barrier to tolerance induction. Purified CD4 $4^{+}$, $\mathrm{CD}^{+}$, or whole T cells were isolated from sensitized mice and transferred to naive congenic mice. Mice receiving either naive selected cells (data not shown) or CD4 ${ }^{+}$ T cells (filled diamonds) from sensitized donors developed high-level donor cell chimerism and accepted donor-type skin grafts indefinitely. The transfer of CD8 ${ }^{+}$ T cells (filled triangles) from sensitized animals prevented tolerance induction in a similar fashion to transferred whole T cells (filled squares) (MST, 21 days). (c) $\mathrm{CD}^{+}$central memory $T$ cells promote rejection more efficiently than "effector" memory T cells. Equal numbers of $\mathrm{CD}^{+}$(open squares), $\mathrm{CD} 8^{+} \mathrm{CD} 62 \mathrm{~L}^{\text {hi }}$ (filled squares), or $\mathrm{CD}^{+} \mathrm{CD}^{2} \mathrm{~L}^{\text {lo }}$ (open triangles) antigen-specific memory $\mathrm{T}$ cells were transferred to naive congenic hosts, which were then subjected to the tolerance protocol (control naive $\mathrm{CD} 8^{+} \mathrm{T}$ cells, filled triangles). Both unseparated $\mathrm{CD} 8^{+}$ memory $T$ cells and central $C D 8^{+}$memory $T$ cells efficiently rejected allogeneic skin grafts in less than 3 weeks (MST, 21 and 18 days, respectively). In contrast, effector memory $T$ cells of the traditional CD62 $\mathrm{L}^{10}$ memory phenotype were less effective at promoting rejection (MST, more than 70 days; $P<0.01$ ).

(data not shown), sensitized (more than 60 days after primary BALB/c skin transplant) B cell-deficient recipients were refractory to tolerance induction and universally rejected their secondary donor allografts by three weeks (MST, 19 days) (data not shown). These results suggested that donor-specific memory $\mathrm{T}$ cells could abrogate the ability of the costimulation blockade-based protocol to induce mixed chimerism and tolerance.

A critical threshold of donor-specific memory T cells is required to resist tolerance induction. To ascertain whether a critical number of memory cells was needed to prevent tolerance induction, we developed an adoptive transfer system in which graded doses of $\mathrm{T}$ cells from sensitized wild-type B6.SJL (CD45.1) mice (skin grafted 60 days earlier) were transferred to naive congenic B6 (CD45.2) recipients before administration of the tolerance induction protocol. Before transfer, the number of donor-reactive $\mathrm{CD}^{+}{ }^{+}$ and $\mathrm{CD}^{+}$memory cells was quantified by intracellular staining for IFN- $\gamma$. Preliminary studies indicated that approximately $1 \%$ of $\mathrm{CD}^{+}$and approximately $2-4 \%$ of $\mathrm{CD}^{+}$splenic $\mathrm{T}$ cells possessed a memory phenotype (production of IFN- $\gamma$ upon short-term in vitro restimulation [4-5 hours]). Two days after the transfer of cells, we then subjected the recipients to the tolerance induction protocol. Mice receiving $\mathrm{T}$ cells from naive congenic hosts were nearly uniformly (over 95\%) susceptible to chimerism and tolerance induction. Resistance to tolerance was observed in recipients reconstituted with $\mathrm{T}$ cells purified from sensitized mice. Groups receiving lower doses of allo-specific memory cells followed a pattern similar to naive mice, with a slightly diminished susceptibility to tolerance induction. In contrast, the majority of mice receiving more than $5 \times 10^{6}$ whole $\mathrm{T}$ cells from sensitized hosts (more than 100,000 memory cells) failed to generate donor cell chimerism and rejected skin grafts by 1 month (Figure 2a). $C D 8^{+}$central memory $T$ cells are the principal mediators of rejection. In additional studies, we investigated the relative contributions of $\mathrm{CD}^{+}$and $\mathrm{CD}^{+}$memory cells in promoting rejection. Equal numbers of purified $\mathrm{CD}^{+}$ or $\mathrm{CD}^{+} \mathrm{T}$ cells from sensitized animals were transferred to naive congenic recipients before tolerance induction $\left(\mathrm{CD}^{+}\right.$and $\mathrm{CD}^{+}$cell preparations were at least $90 \%$ pure with no more than $1 \%$ contamination of the complementary subset). Animals receiving $\mathrm{CD}^{+}$memory $\mathrm{T}$ cells quickly rejected their skin grafts, similar to animals receiving unfractionated memory cells (MST, 21 days). In stark contrast, animals that received $\mathrm{CD}^{+}$memory cells developed donor cell chimerism and enjoyed prolonged graft survival (more than 100 days) (Figure $2 \mathrm{~b}$ ). These data suggest that $\mathrm{CD}^{+}$ memory cells represent a powerful barrier to tolerance induction in this model, and additional pathways, crucial for memory cell activation and function, need to be identified to allow for successful tolerance induction in the face of existing donor-specific $\mathrm{CD}^{+} \mathrm{T}$ cells.

Recently, there have been several reports documenting the phenotypic and functional heterogeneity that exists in the memory $\mathrm{T}$ cell compartment (2-4). A model of central and effector memory has been proposed because of differences in location and expression of homing molecules (CD62L and CCR7) (2). Central memory T cells $\left(\mathrm{CD} 62 \mathrm{~L}^{\text {hi }} \mathrm{CCR}^{+}\right)$lack immediate "killing" activity but rapidly produce IL-2 when restimulated in vitro, suggesting a greater proliferative capacity than their effector counterparts. Effector memory T cells $\left(\mathrm{CD} 62 \mathrm{~L}^{\text {lo }}\right.$ CCR7-), on the other hand, rapidly produce IFN- $\gamma$, contain intracellular perforin, and are capable of immediate ex vivo cytotoxicity. Given their capability for immediate 
effector function and favorable homing properties, it has been suggested that effector memory $\mathrm{T}$ cells would be better able to control subsequent immune challenges and that central memory $T$ cells remain available in secondary lymphoid organs to provide "help" and to replenish dwindling effectors (1). In order to investigate the roles of effector and central memory $\mathrm{T}$ cells in preventing tolerance induction in our model, we transferred equal numbers $(200,000)$ of antigen-specific (as assessed by IFN- $\gamma$ production upon short-term restimulation in vitro) $\mathrm{CD}^{+}, \mathrm{CD}^{+} \mathrm{CD}^{2} 2 \mathrm{~L}^{\text {hi }}$, or $\mathrm{CD} 8^{+} \mathrm{CD} 62^{\text {lo }}$ memory $\mathrm{T}$ cells into naive congenic mice. We then subjected each group to the tolerance induction protocol as described above. As in previous experiments, mice receiving unfractionated $\mathrm{CD}^{+}$memory $\mathrm{T}$ cells quickly rejected donor allografts (MST, 21 days). Surprisingly, despite the lack of immediate cytotoxic potential, central $\mathrm{CD}^{+}$memory $\mathrm{T}$ cells promoted rejection more efficiently (MST, 18 days) than effector $\mathrm{CD}^{+}$memory $\mathrm{T}$ cells (MST, more than 70 days; $P<0.01$ ) (Figure $2 c$ ).

Virally induced donor-specific memory cells prevent tolerance induction. Given the observations that viral infections induce alloreactive memory responses and that donorspecific memory can prevent tolerance, we established a model to evaluate the impact of virally induced allo-specific memory on tolerance induction. We hypothesized that although a single infection with LCMV may generate a sufficient heterologous response at the peak of the antiviral response to induce graft rejection, the number of alloreactive cells that remain in the memory phase may be insufficient to overcome the tolerance protocol. Furthermore, infection of a specific pathogen-free mouse with a single virus may not adequately simulate the clinical situation in which exposure to a myriad of pathogens and antigens is the rule and memory cells typically represent $40-50 \%$ of the $T$ cell pool in adult humans (26). Thus, we studied animals that received multiple, distinct viral infections over time to more closely approximate the acquired immune history of human patients or outbred nonhuman primates, which commonly show evidence of exposure to many pathogens (e.g., EBV, CMV, VZV, polyoma). Accordingly, we infected mice (C57BL/6) with either a single pathogen (LCMV, VV, or VSV) or sequentially with multiple pathogens (LCMV $\rightarrow \mathrm{VV}$ or $\mathrm{LCMV} \rightarrow \mathrm{VV} \rightarrow \mathrm{VSV}$ ), allowing 6-8 weeks between infections and at least 8 weeks after the final infection before study in the tolerance experiments to ensure that only memory cells remained. Uninfected, age-matched naive $\mathrm{B} 6$ mice uniformly developed high-level chimerism (mean chimerism, $49.1 \pm 4.22$ in 10 of 10 animals) and accepted donor skin grafts indefinitely (10 of 10; MST, more than 100 days) (Figure 3a). Mice that were immune to a single pathogen (LCMV, Figure 3 , and VV, data not shown) demonstrated a slight decrease,

\section{Figure 3}

although not statistically significant in this experiment, in the number of animals becoming chimeric and tolerant (9 of 12, $P=0.2$ when compared with the naive group). This slightly diminished susceptibility to tolerance mirrored our earlier results when suboptimal doses of memory cells were transferred (Figure 2a). In other experiments, tolerance was induced in 37 of $45 \mathrm{LCMV}$ immune mice (82\%), which is lower than the over $95 \%$ tolerance induction rate in naive mice, as observed in well over 200 nonconcurrent control recipients, suggesting that prior exposure to a potent infection such as LCMV may indeed have a moderate effect. In contrast to uninfected recipients, mice that had previously been exposed to multiple viral infections were refractory to tolerance induction and rejected their allografts (MST, 24 days; $n=12$ ) (Figure 3a). In all groups, the presence of stable donor hematopoietic cell engraftment correlated with long-term skin graft survival (i.e., no chimerism indicated no skin graft survival) (Figure 3b). Not surprisingly, the number of virally induced alloreactive memory cells was higher in animals with multiple infections than in singly infected animals (data not shown).

$D S G$, an inhibitor of NF- $\kappa B$ translocation, promotes tolerance of antigen-specific memory Tcells. Although naive cells require stimulation through the $\mathrm{T}$ cell receptor and additional costimulatory signals for their activation, memory $T$ cells are less stringent in their activation requirements. In an attempt to promote tolerance of antigen-specific memory $T$ cells, we identified potential pathways that may inhibit memory cell activation. AntiCD25, rapamycin, and blocking monoclonal antibodies against the common $\gamma$ chain have all been reported to act synergistically with costimulation blockade to inhibit allograft rejection (27-29). In addition, we examined the effects of the novel immunosuppressant DSG, which has shown efficacy in both nonhuman primate models and human patients $(30,31)$. DSG is reported

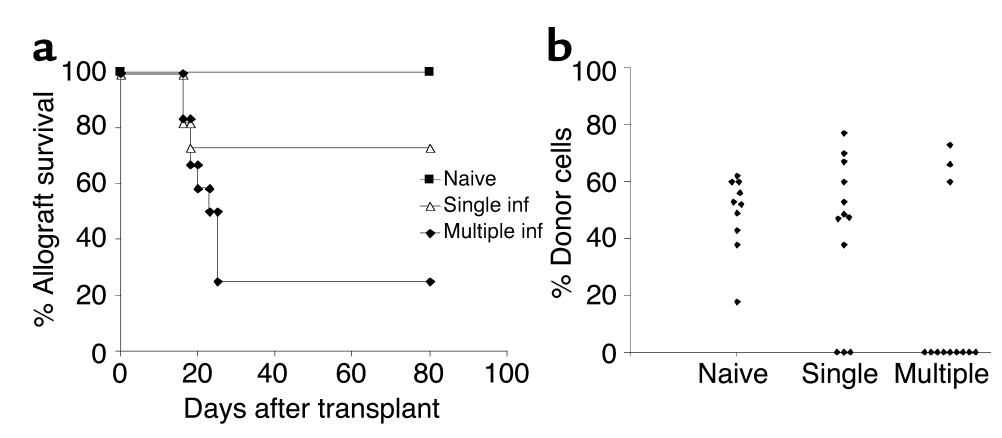

Virally induced, alloreactive memory cells prevent tolerance induction. Naive, monoimmune ( $\mathrm{LCMV}$ or $\mathrm{W}$ ), or polyimmune mice ( $\mathrm{LCMV} \rightarrow \mathrm{W}$ or $\mathrm{LCMV} \rightarrow \mathrm{W} \rightarrow \mathrm{VSV}$ ) received the mixed chimerism, costimulation blockade-based tolerance protocol. (a and b) Skin graft survival and donor chimerism levels after tolerance induction. Naive mice universally accept donor-type skin allografts (left panel, filled squares) and become high-level mixed chimeras (10 of 10 , right panel). The majority of mice immune to a single pathogen become tolerant, although not as consistently as naive mice ( 9 of 12 , open triangles). Mice immune to multiple pathogens are refractory to tolerance induction ( 3 of 12; MST, 24; $P=0.002$ when compared with naive mice; filled diamonds). Inf, infection(s). 
a

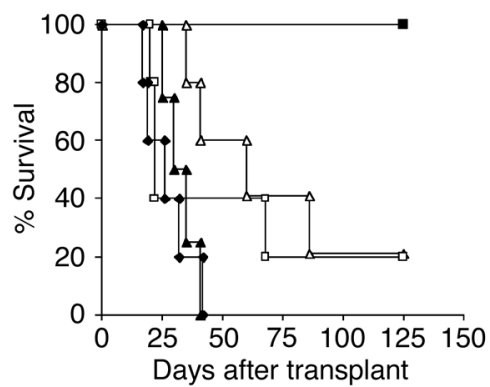

b

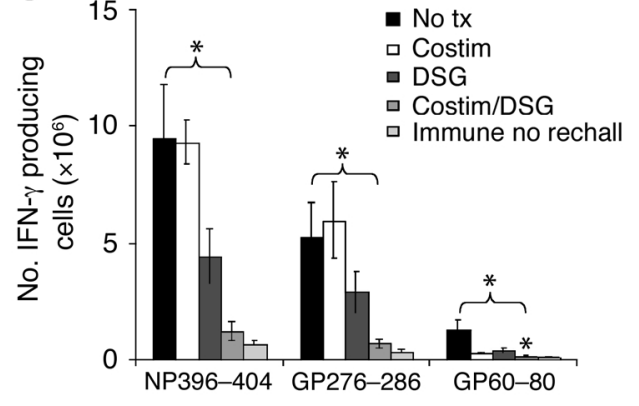

Figure 4

DSG and costimulation blockade synergistically inhibit memory cells. Both alloreactive and virus-specific memory cell responses were analyzed. (a) Skin graft survival of mice that had previously ( 1 week earlier) received T cells from sensitized congenic mice and were treated with either the tolerance induction protocol alone or in combination with various agents. The combination of DSG and costimulatory blockade promoted tolerance of donor-specific memory cells ( $n=5$; MST, 100 days; filled squares). The costimulation blockade tolerance regimen given alone ( $n=5$, open squares) or in combination with other agents, including rapamycin $(n=5$, filled triangles), anti-CD25 ( $n=5$, open triangles), and anti- $\gamma_{c}(n=5$, filled diamonds) failed to inhibit memory-dependent rejection. Two additional experiments demonstrated similar results. (b) LCMV-immune mice were rechallenged with LCMV clone 13 and treated with costimulation blockade (costim), DSG, or the combination of costimulation blockade and DSG. Five days after rechallenge, $\mathrm{CD}^{+}$(class I restricted NP396-404 and GP276-286) and CD4 ${ }^{+}$(class II restricted P13 GP60-80) responses were analyzed. The combination of costimulatory blockade and DSG synergistically inhibited LCMV-specific CD8 ${ }^{+}$memory T cells $\left({ }^{*} P<0.01\right)$. No tx, no treatment; immune no rechall, immune mouse without viral rechallenge.

In order to more fully evaluate the effects of costimulation blockade and DSG on memory cell activation and function, we also studied recall responses in the LCMV model. Mice immune to LCMV (LCMV infection more than 60 days earlier) were rechallenged with LCMV and were administered costimulation blockade (CTLA4-Ig/ anti-CD40L) alone, DSG alone, costimulation blockade and DSG, or no treatment. Five days after rechallenge, $\mathrm{T}$ cells were harvested and epitope-specific $\mathrm{CD}^{+}$ (class I restricted NP396-404 and GP276-286) and $\mathrm{CD}^{+}$ (class II restricted P13 GP61-80) memory responses were analyzed. Memory responses in mice treated with costimulation blockade were similar to those in immune control animals receiving no treatment (Figure 4b), consistent with studies demonstrating that memory $\mathrm{T}$ cells do not require costimulation delivered through CD28 or CD40L

to bind to the intracellular chaperone Hsc70 and inhibit translocation of NF- $\kappa \mathrm{B}$, a transcription factor important for $\mathrm{T}$ cell activation and survival $(32,33)$.

Using our adoptive transfer model, we evaluated the agents for their ability to inhibit memory cell activation. Mice that had previously received T cells from sensitized congenic mice (approximately 200,000 memory $\mathrm{T}$ cells) were subjected to the tolerance protocol alone or the tolerance protocol in combination with the specific inhibitors. The addition of rapamycin, anti-CD25, or anti- $\gamma_{c}$ failed to promote tolerance of anti-donor memory cells. The mice rejected their grafts at similar times as control animals receiving the tolerance protocol alone (Figure 4a), suggesting that memory cell activation and function was not critically dependent on those pathways. Interestingly, mice receiving the costimulation blockade-based tolerance protocol and DSG uniformly became tolerant and accepted their allografts indefinitely despite prior transfer of antidonor memory cells ( $n=5$; MST, more than 100 days) (Figure 4a). Upon further follow-up, animals receiving the tolerance protocol and DSG accepted skin allografts and maintained stable donor chimerism for at least 1 year. When rechallenged with secondary donor and third-party skin allografts, animals accepted secondary donor skin grafts while rejecting third-party grafts in control time, suggesting that the combination of costimulation blockade and DSG effectively promoted tolerance of both naive and memory donor-reactive $\mathrm{T}$ cells (data not shown). for their activation. DSG alone exerted only a moderate effect on the antiviral memory response, whereas the combination of costimulation blockade and DSG synergistically impaired the antiviral memory response - in particular, the $\mathrm{CD}^{+}$memory $\mathrm{T}$ cell response (Figure $4 \mathrm{~b})(P=0.01$ when compared with no treatment).

To determine if the combination of costimulation blockade and DSG promoted tolerance of memory cells in an antigen-specific manner, we transferred memory cells from LCMV-immune mice (approximately 500,000 P14 memory $\mathrm{T}$ cells) and from allo-immune mice (approximately 400,000 donor-specific memory $\mathrm{T}$ cells). Two days later, we administered the tolerance protocol alone or in combination with DSG (Figure 5a). Similar to previous results, mice receiving the tolerance protocol and DSG accepted donor-specific skin grafts, indicating effective inhibition of the transferred allo-memory cells. Animals receiving the tolerance protocol alone rejected grafts by 1 month ( $n=5$; MST, 28 days) (data not shown). To test whether the effect of DSG was antigen specific, we assessed the functional response of the transferred memory cells in both groups 2 months after transplantation. As expected, mice treated with the protocol and DSG failed to display any donor-reactive $\mathrm{T}$ cells (Figure $5 \mathrm{~b}$ ), indicating that the regimen had effectively tolerized the transferred allo-specific memory cells. Conversely, mice treated with the costimulation blockade tolerance protocol alone clearly exhibited the presence of donor-reactive memory cells (Figure 5b). All of the IFN- $\gamma^{+}$cells resided in the transferred population $\left(\mathrm{CD} 45.1^{+}\right)$(data not 
a

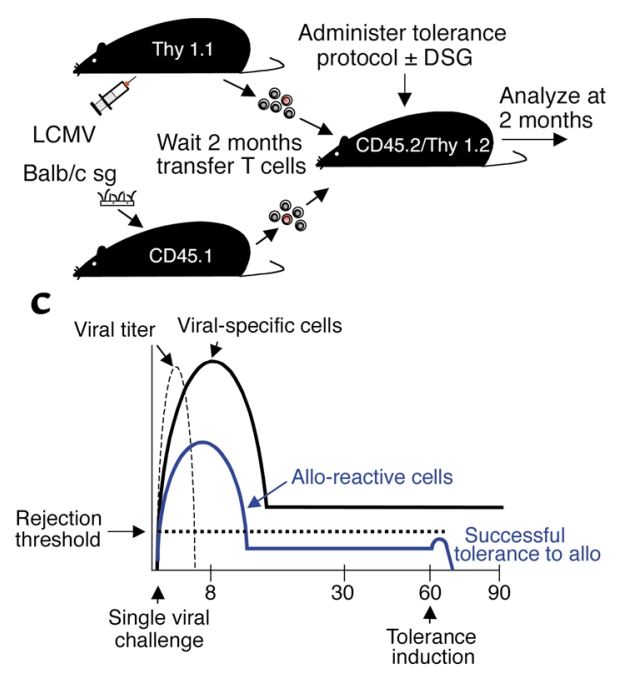

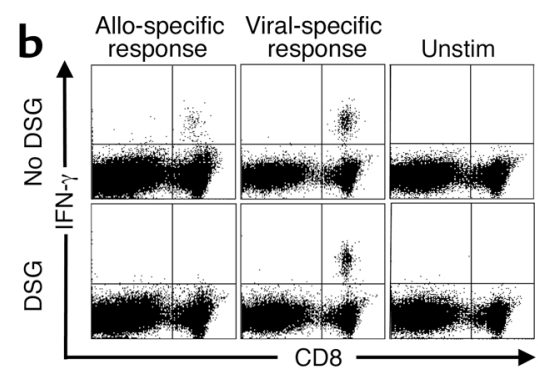

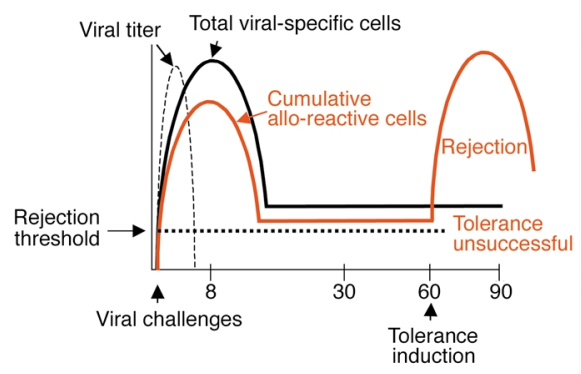

Figure 5

Antigen-specific tolerance of memory cells. (a) Congenic mice were either infected with LCMV (Thy 1.1) or received a skin allograft (CD45.1). Eight weeks after challenge, T cells were isolated to obtain both allo- and virus-specific memory cells and then transferred into naive B6 mice (CD45.2/Thy1.2). Mice then received the tolerance protocol or the tolerance protocol with DSG. sg, skin graft. (b) When the tolerance protocol alone was administered, both alloreactive and virus-specific memory cell populations were preserved, resulting in allograft rejection. When given in combination with DSG, donor-reactive cells were specifically deleted, and viral memory remained intact upon rechallenge. Unstim, unstimulated. (c) Model for heterologous immunity as a barrier to transplantation tolerance.

shown), suggesting that the tolerance protocol effectively inhibited the generation of new effectors but failed to control pre-existing memory cells. Importantly, both groups displayed similar numbers of functional, virusspecific memory $\mathrm{T}$ cells, indicating that the combination of costimulation blockade and DSG acts synergistically to impair memory $\mathrm{T}$ cell responses in an antigen-specific manner, whereas pre-existing memory cells, which are not engaged by their cognate antigen during the treatment period, are preserved for future challenges.

\section{Discussion}

Memory is an indispensable component of the immune response. The primary purpose of the amnestic response is to protect the host from future challenges of the identical pathogen. There is also accumulating evidence to suggest that in certain settings, memory elicited by certain viruses can enhance the clearance of even unrelated pathogens (5). Unfortunately, the heterologous response may also lead to immune-mediated disease in some circumstances $(34,35)$. Another potentially adverse consequence of an antiviral response is the production of effector $\mathrm{T}$ cells capable of directly recognizing foreign $\mathrm{MHC}$ and the subsequent formation of long-lived alloreactive memory cells.

An unresolved area of intense interest in the field of transplantation biology has been the somewhat puzzling and recurring observation that tolerance strategies, which promote long-lived graft survival in rodents, have consistently failed when evaluated in nonhuman primate models and clinical trials. This has led to the emerging concept that environmental exposures and large memory pools may constitute a more formidable barrier to tolerance induction than the immune system of experimental rodents.

Although cross-reactivity of pathogen-reactive $\mathrm{T}$ cells with allogeneic MHC complexes has been demonstrated at the cellular level, our results provide direct in vivo evidence that prior immune encounters can provide a powerful barrier to tolerance induction. Viral infections lead to the generation of alloreactive memory $T$ cells, which in turn confer resistance to tolerance induction. Whether this is the result of bystander activation, mimicry on a molecular level (viral peptide bound by self-MHC resembling self-peptide bound by foreign MHC) or a combination of the two remains to be fully elucidated $(36,37)$. The number of memory cells the host has accumulated is critical; as we have shown, there is a threshold of memory cells necessary to promote rejection. Although naive animals and those receiving a single infection were susceptible to tolerance induction, it is very likely that these animals have encountered far fewer pathogens than potential transplant recipients, in whom the prevalence of viral infections (including EBV, CMV, HSV, VSV, influenza, etc.), as in the general population, is quite high. In support of our hypothesis, we have shown that animals immune to multiple viruses, perhaps a closer approximation to "immune-experienced" hosts, almost universally fail to become tolerant when using a regimen that uniformly leads to tolerance in naive recipients.

Our results indicate that $\mathrm{CD} 8^{+}$memory $\mathrm{T}$ cells are the principal mediators of rejection in this model. However, this does not necessarily exclude a role for $\mathrm{CD}^{+}$memory as a barrier to tolerance induction in other settings. In 
this model, the number of $\mathrm{CD} 4^{+}$memory $\mathrm{T}$ cells generated in vivo and then transferred to naive hosts was approximately fourfold lower than the number of memory $\mathrm{CD}^{+} \mathrm{T}$ cells. Thus, in this system, the number of $\mathrm{CD}^{+}$memory $\mathrm{T}$ cells may not achieve the critical threshold needed to precipitate rejection.

Perhaps one of the most interesting new areas of investigation in immunology is the description of functional subsets within the memory $\mathrm{T}$ cell pool. Although the properties of these subsets have not yet been completely defined, one might have anticipated that effector memory cells, which are programmed to home to peripheral locations and primed for immediate effector function, would constitute a more formidable barrier to tolerance than their central memory counterparts. Interestingly, our data indicate that central memory $\mathrm{T}$ cells are actually considerably more potent on a per-cell basis in their ability to resist tolerance induction. This suggests that central and effector memory may differ in dependence of each on CD28 and CD40 costimulatory signals or that the superior proliferative capacity of central memory cells is necessary to resist tolerance.

Although the signal requirements for memory cell activation and function have not been completely defined, traditional costimulatory signals (e.g., CD28/B7, CD40/ CD40L) do not seem to be essential $(15,16)$. Recent work has identified cytokines (IL-15 and IL-7) important for maintenance of memory through homeostatic proliferative mechanisms $(35,38-40)$. Interestingly, neither of these cytokines was essential for productive recall responses. Consistent with these data, we present data to suggest that the interruption of key cytokine signaling pathways, using either blocking antibodies (anti-IL-2R or anti- $\gamma_{c}$ ) or the immunosuppressive molecule rapamycin, did not significantly inhibit memory cell function in our model. In contrast, the combination of $T$ cell costimulation blockade and the immunosuppressive agent DSG synergized to inhibit the function of memory $\mathrm{T}$ cells in both allograft and viral models. The putative mechanism of action of DSG is to inhibit translocation of NF- $\mathrm{kB}$, a transcription factor involved in many cellular processes, including $T$ cell activation (41). Interestingly, NF- $\mathrm{KB}$ plays a critical role in determining the balance between pro- and antiapoptotic factors, which in turn determine the fate of the cell $(42,43)$. The experiments in which we cotransfer LCMV-specific and allospecific memory cells indicate that the combination of DSG and costimulation blockade can induce inactivation of antigen-specific memory cells, since only those cells encountering their cognate ligands during the period of drug administration were affected. Protective immunity was preserved, since viral memory cells not specific for the tolerogen (donor marrow) remained fully competent and capable of responding to a subsequent viral rechallenge. Inhibition of NF- $\mathrm{KB}$ at a key step in memory $\mathrm{T}$ cell activation may result in a decrease in essential cellular survival signals and tip the balance toward cell death, permitting the deletion of antigenspecific memory cells.
Our data suggest that heterologous immunity, in particular virally induced alloreactive memory, may represent an underexplored barrier to tolerance induction in transplantation. It provides a potential explanation for the lack of success when tolerance regimens, effective in rodent models, are tested in nonhuman primate models or in human patients. Although cross-reactive responses between unrelated viruses have been documented, simple probability suggests that the occurrence of heterologous allogeneic responses after pathogen exposure should be even more likely (given the high precursor frequency of the direct allogeneic response).

There are three distinct possibilities whereby pathogen exposure could influence the host's response to allo-antigen. First there may be an active, ongoing infection at the time of tolerance induction. This includes exposure to an acute infection coincidental with attempts at tolerance or the presence of a smoldering persistent, productive infection in which the pathogen is present but under immune control. Potential unfavorable outcomes include the possibility of tolerance to the pathogen and loss of immune control, or the ongoing antiviral response may promote rejection and an inability to induce tolerance. We and others have attempted to address this question experimentally and in prior studies have demonstrated that a concurrent acute infection with LCMV at the time of tolerance induction can precipitate rejection $(36,37)$. Recently, we have also demonstrated that the presence of an ongoing, persistent productive viral infection interferes with attempts to induce tolerance (44). Heeger and colleagues have recently published similar findings for the chronic parasitic infection Leishmania (45). Both of these studies address a similar question in systems in which there is eventual immune control of the infection but the pathogen is never completely cleared and persists for the life of the mouse $(18,46,47)$. This may be of particular relevance to a subset of transplant patients who suffer from ongoing, chronic infections such as hepatitis C. However, mechanisms in addition to simple cross-reactivity may also be important in these models. For example, the active immune response against the persistent pathogen may in and of itself provide a means, such as elevated levels of cytokines and growth factors, to overcome costimulatory blockade.

The second scenario is exposure of the host to multiple acute viral infections that resolve completely before attempts of tolerance are initiated. This condition, which is present in virtually all human transplant recipients, has not previously been investigated experimentally. In this work, we examined the influence of prior resolved infections on the host's memory pool and demonstrated dramatic effects on tolerance induction. Our results indicate that a single prior acute viral infection (e.g., LCMV, VV), which is subsequently completely cleared, is inadequate to generate a sufficient number of alloreactive memory $\mathrm{T}$ cells necessary to reach the critical threshold required to impair attempts at tolerance and promote rejection. Humans, unlike experimental rodents, have been exposed to a wide variety of pathogens, each 
of which has the potential to generate alloreactive memory (Figure 5c). Our data indicate that if sufficient numbers of donor-specific memory $T$ cells accumulate after multiple acute viral infections over time, a critical threshold is exceeded, and attempts at tolerance induction that fail to target memory cells will be unsuccessful (Figure 5c). Lastly, the influence of pathogens that are harbored in a quiescent, true latent state has yet to be tested experimentally. Ongoing studies in our laboratory seek to address this important issue.

In conclusion, while further work is necessary to identify the pathways critical for memory cell activation and survival, the combination of costimulation blockade and DSG offers a clinically relevant strategy to promote antigen-specific tolerance of environmentally induced, alloreactive memory $T$ cells. Thus, future plans for tolerance trials in humans should take into consideration that even recipients of primary transplants may not be as "naive" as practitioners believe.

\section{Acknowledgments}

This work was supported in part by research grants DK/AI40519, CA74364-03, and AI44644 from the National Institutes of Health; by the Engineering Research Center Program of the National Science Foundation under award number EEC-9731643; and by the Carlos and Marguerite Mason Trust.

1. Kaech, S.M., Wherry, E.J., and Ahmed, R. 2002. Effector and memory T-cell differentiation: implications for vaccine development. Nat. Rev. Immunol. 2:251-262.

2. Sallusto, F., Lenig, D., Forster, R., Lipp, M., and Lanzavecchia, A. 1999. Two subsets of memory $\mathrm{T}$ lymphocytes with distinct homing potentials and effector functions. Nature. 401:708-712.

3. Masopust, D., Vezys, V., Marzo, A.L., and Lefrancois, L. 2001. Preferential localization of effector memory cells in nonlymphoid tissue. Science. 291:2413-2417.

4. Reinhardt, R.L., Khoruts, A., Merica, R., Zell, T., and Jenkins, M.K. 2001

Visualizing the generation of memory CD $4 \mathrm{~T}$ cells in the whole body. Nature. 410:101-105.

5. Welsh, R.M., and Selin, L.K. 2002. No one is naive: the significance of heterologous T-cell immunity. Nat. Rev. Immunol. 2:417-426.

6. Blattman, J.N., et al. 2002. Estimating the precursor frequency of naive antigen-specific CD8 T cells. J. Exp. Med. 195:657-664.

7. Suchin, E.J., et al. 2001. Quantifying the frequency of alloreactive T cells in vivo: new answers to an old question. J. Immunol. 166:973-981.

8. Mason, D. 1998. A very high level of crossreactivity is an essential feature of the T-cell receptor. Immunol. Today. 19:395-404.

9. Sheil, J.M., Bevan, M.J., and Lefrancois, L. 1987. Characterization of dualreactive $\mathrm{H}-2 \mathrm{~Kb}$-restricted anti-vesicular stomatitis virus and alloreactive cytotoxic T cells. J. Immunol. 138:3654-3660.

10. Yang, H., and Welsh, R.M. 1986. Induction of alloreactive cytotoxic T cells by acute virus infection of mice. J. Immunol. 136:1186-1193.

11. Braciale, T.J., Andrew, M.E., and Braciale, V.L. 1981. Simultaneous expression of $\mathrm{H}$-2-restricted and alloreactive recognition by a cloned line of influenza virus-specific cytotoxic T lymphocytes. J. Exp. Med. 153:1371-1376.

12. Burrows, S.R., Khanna, R., Burrows, J.M., and Moss, D.J. 1994. An alloresponse in humans is dominated by cytotoxic T lymphocytes (CTL) crossreactive with a single Epstein-Barr virus CTL epitope: implications for graft-versus-host disease. J. Exp. Med. 179:1155-1161.

13. Maruya, E., Takemoto, S., and Terasaki, P.I. 1993. HLA matching: identification of permissible HLA mismatches. Clin. Transpl. 9:511-520.

14. Heeger, P.S., et al. 1999. Pretransplant frequency of donor-specific, IFNgamma-producing lymphocytes is a manifestation of immunologic memory and correlates with the risk of posttransplant rejection episodes. J. Immunol. 163:2267-2275.

15. Lanzavecchia, A., and Sallusto, F. 2000. Dynamics of T lymphocyte responses: intermediates, effectors, and memory cells. Science. 290:92-97.

16. London, C.A., Lodge, M.P., and Abbas, A.K. 2000. Functional responses and costimulator dependence of memory $\mathrm{CD} 4^{+} \mathrm{T}$ cells. J. Immunol. 164:265-272.
17. Valujskikh, A., Pantenburg, B., and Heeger, P.S. 2002. Primed allospecific T cells prevent the effects of costimulatory blockade on prolonged cardiac allograft survival in mice. Am. J. Transplant. 2:501-509.

18. Ahmed, R., Salmi, A., Butler, L.D., Chiller, J.M., and Oldstone, M.B. 1984. Selection of genetic variants of lymphocytic choriomeningitis virus in spleens of persistently infected mice. Role in suppression of cytotoxic $\mathrm{T}$ lymphocyte response and viral persistence. J. Exp. Med. 160:521-540.

19. Adams, A.B., et al. 2001. Costimulation blockade, busulfan, and bone marrow promote titratable macrochimerism, induce transplantation tolerance, and correct genetic hemoglobinopathies with minimal myelosuppression. J. Immunol. 167:1103-1111.

20. Harrington, L.E., Whitton, J.L., and Ahmed, R. 2002. Recombinant vaccinia virus-induced T-cell immunity: quantitation of the response to the virus vector and the foreign epitope. J. Virol. 76:3329-3337.

21. Murali-Krishna, K., et al. 1998. Counting antigen-specific CD8 T cells: a reevaluation of bystander activation during viral infection. Immunity. 8:177-187.

22. Lau, L.L., Jamieson, B.D., Somasundaram, T., and Ahmed, R. 1994. Cytotoxic T-cell memory without antigen. Nature. 369:648-652.

23. Kundig, T.M., et al. 1996. On the role of antigen in maintaining cytotoxic T-cell memory. Proc. Natl. Acad. Sci. U. S. A. 93:9716-9723.

24. Whitmire, J.K., and Ahmed, R. 2000. Costimulation in antiviral immunity: differential requirements for CD4(+) and CD8(+) T cell responses. Curr. Opin. Immunol. 12:448-455.

25. Butz, E.A., and Bevan, M.J. 1998. Massive expansion of antigen-specific CD8+ T cells during an acute virus infection. Immunity. 8:167-175.

26. Douek, D.C., et al. 1998. Changes in thymic function with age and during the treatment of HIV infection. Nature. 396:690-695.

27. Li, Y., et al. 1999. Blocking both signal 1 and signal 2 of T-cell activation prevents apoptosis of alloreactive $\mathrm{T}$ cells and induction of peripheral allograft tolerance. Nat. Med. 5:1298-1302.

28. Jones, T.R., et al. 2002. The role of the IL-2 pathway in costimulation blockade-resistant rejection of allografts. J. Immunol. 168:1123-1130.

29. Demirci, G., et al. 2002. On CD28/CD40 ligand costimulation, common gamma-chain signals, and the alloimmune response. J. Immunol. 168:4382-4390.

30. Bartholomew, A.M., et al. 1999. Tolerance in a concordant nonhuman primate model. Transplantation. 68:1708-1716.

31. Knechtle, S.J. 2001. Treatment with immunotoxin. Philos. Trans. R. Soc. Lond. B Biol. Sci. 356:681-689.

32. Nadler, S.G., Tepper, M.A., Schacter, B., and Mazzucco, C.E. 1992. Interaction of the immunosuppressant deoxyspergualin with a member of the Hsp70 family of heat shock proteins. Science. 258:484-486.

33. Tepper, M.A., Nadler, S.G., Esselstyn, J.M., and Sterbenz, K.G. 1995. Deoxyspergualin inhibits kappa light chain expression in $70 Z / 3$ pre-B cells by blocking lipopolysaccharide-induced NF-kappa B activation. J. Immunol. 155:2427-2436.

34. Selin, L.K., Varga, S.M., Wong, I.C., and Welsh, R.M. 1998. Protective heterologous antiviral immunity and enhanced immunopathogenesis mediated by memory T cell populations. J. Exp. Med. 188:1705-1715.

35. Chen, H.D., et al. 2001. Memory $\mathrm{CD8}^{+} \mathrm{T}$ cells in heterologous antiviral immunity and immunopathology in the lung. Nat. Immunol. 2:1067-1076.

36. Tan, J.T., et al. 2002. Interleukin (IL)-15 and IL-7 jointly regulate homeostatic proliferation of memory phenotype $\mathrm{CD}^{+}$cells but are not required for memory phenotype CD4 ${ }^{+}$cells. J. Exp. Med. 195:1523-1532.

37. Welsh, R.M., et al. 2000. Virus-induced abrogation of transplantation tolerance induced by donor-specific transfusion and anti-CD154 antibody. J. Virol. 74:2210-2218.

38. Williams, M.A., et al. 2001. Characterization of virus-mediated inhibition of mixed chimerism and allospecific tolerance. J. Immunol. 167:4987-4995.

39. Goldrath, A.W., et al. 2002. Cytokine requirements for acute and basal homeostatic proliferation of naive and memory $\mathrm{CD}^{+} \mathrm{T}$ cells. J. Exp. Med. 195: $1515-1522$

40. Becker, T.C., et al. 2002. Interleukin 15 is required for proliferative renewal of virus-specific memory CD8 T cells. J. Exp. Med. 195:1541-1548.

41. Schluns, K.S., Williams, K., Ma, A., Zheng, X.X., and Lefrancois, L. 2002. Cutting edge: requirement for IL-15 in the generation of primary and memory antigen-specific CD8 T cells. J. Immunol. 168:4827-4831.

42. Karin, M., and Lin, A. 2002. NF-kappaB at the crossroads of life and death. Nat. Immunol. 3:221-227.

43. Beg, A.A., and Baltimore, D. 1996. An essential role for NF-kappaB in preventing TNF-alpha-induced cell death. Science. 274:782-784.

44. Williams, M.A., et al. 2002. Cutting edge: Persistent viral infection prevents tolerance induction and escapes immune control following CD28/CD40 blockade-based regimen. J. Immunol. 169:5387-5391.

45. Pantenburg, B., Heinzel, F., Das, L., Heeger, P.S., and Valujskikh, A. 2002. $\mathrm{T}$ cells primed by Leishmania major infection cross-react with alloantigens and alter the course of allograft rejection. J. Immunol. 169:3686-3693.

46. Reiner, S.L., and Locksley, R.M. 1995. The regulation of immunity to Leishmania major. Annu. Rev. Immunol. 13:151-177.

47. Sacks, D., and Noben-Trauth, N. 2002. The immunology of susceptibility and resistance to leishmania major in mice. Nat. Rev. Immunol. 2:845-858. 